\title{
Adaptive Gain Scheduling Based controller for Networking Control Systems
}

\author{
Rasha Shamit Salman ${ }^{1}$, Ahmad T. Abdulsadda ${ }^{1}$ \\ ${ }^{I}$ Department of Communications Technical Engineering, Al-Najaf Technical Engineering College / Najaf, Al-Furat Al-Awsat \\ Technical University ATU, Najaf, 54001, Republic of Iraq \\ E-mail: rashaalhessnawi@gmail.com \\ E-mail: ahmadabdulsadda@yahoo.com
}

\begin{abstract}
Recently, researchers have focused on integrating wireless networks with control systems, which has shown what is called Wireless Network Control Systems (WNCS). On the other hand, these systems face several problems and one of these problems is a time delay. In this paper, the Fuzzy logic with the Proportional -Integral-Derivative (PID) controller is proposed in the WNCS design, the Particle Swarm Optimization (PSO) technique is used to obtain optimum rules. The ZigBee network used to send and receive data between the system control and the controller nodes. The proposed controller aims to reduce the time delays that can make the system stable in the event of an overload on the network. The obtained results showed that the proposed controller when the number of nodes increased by 500 , the system remains stable.
\end{abstract}

Keywords- PID controller, Fuzzy, PSO, WNCS, True Time Tools.

\section{INTRODUCTION}

Wireless technologies have been successful in many industrial applications where Wireless Network Control System (WNCS) has some advantages in terms of cost, great flexibility and ease of maintenance such as in all its parts controllers, sensors, actuators and network [1]. Figure 1 is shown a general network control system that uses in industrial systems. Three challenges in building control systems are communication networks, control theory, and real-time systems [2]. Although the advantages in WNCS, there are still some disadvantages are packet losses, delay, communication security and reliability, limited bandwidth, development of different network protocols, and packet drops [3]. On the other hand, like wireless networks, for example in WNCS, are used for control applications further aspects should be considered. WNCS need not maximize reliability, such as in the monitoring applications [4].

Abdul-Hussain et al.[5] the authors compared the two controllers PID and Fractional Order Proportional Integral-Derivative (FOPID) based on the PSO algorithm in WNCS design to minimize time delays and to keep the system stable. PSO is used to find parameters of PID and FOPID controller and tested the system with various loads. $\mathrm{Wi}-\mathrm{Fi}$ is used as wireless network communication.

Sliding Mode and PID controllers are designed controller by Salman et al. [6] to Control DC-motor speed over the Zigbee network which is a communication network based on the NCS and study test different network scenarios for both controllers, considering different parameters of the Zigbee network.

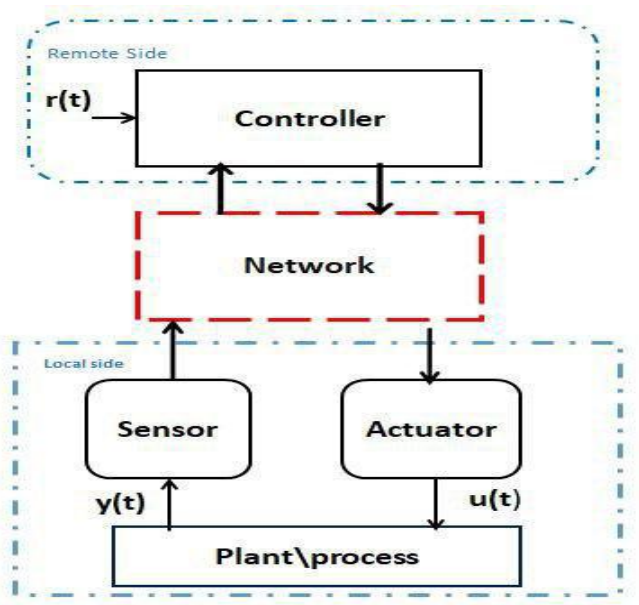

Fig. 1 General Structure of (NCS) [2]

Fuzzy logic designed by Peng et al. [7] to give Tuning parameters into a PID controller with second-order inertia in the plant and the network communication mode is CSMA/CD (Ethernet). Aung et al. [8] ZigBee network Simulated by True-time based on MATLAB / Simulink network that transmitted data between the PID controller and a DC motor through the network.

Awad et al. [8] the authors suggest using the traditional PID controller with fuzzy logic and Gain Scheduling (GS) in the NCS design. A wired network (Ethernet) is used as 


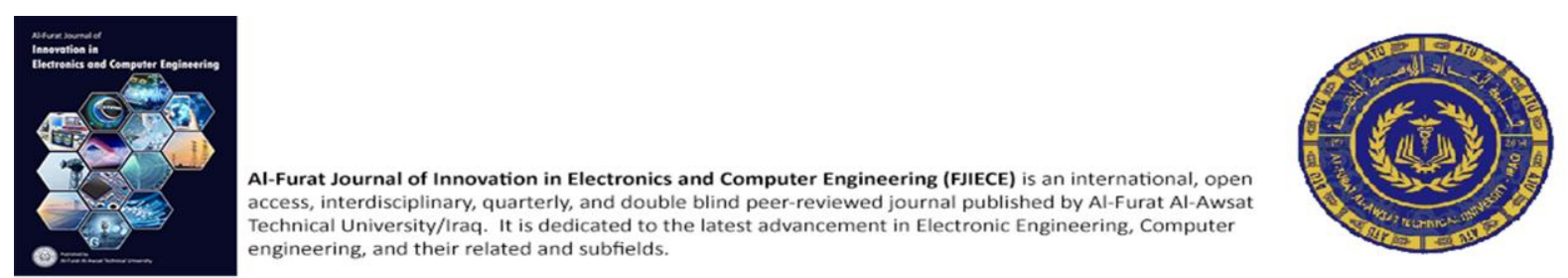

network communication. The F-PID-GS is tested with different load conditions and packet loss randomization. The most important problems facing these systems. One of these problems is a time delay at network overload. In order to keeps system performance and stability, these problems are addressed with a robust controller design. In this paper, the Fuzzy PID controller to control the plant wirelessly is suggested based on the PSO algorithm to find optimal rules for fuzzy logic, ZigBee network is used to link control system parts.

The rest of this paper is structured as follows. Section II explains the controller design. Section III. presents the true time toolbox, Section IV. presents the ZigBee network, while section V. explains the proposed simulation of WNCS, The proposed Fuzzy PID controller and PSO are introduced in section V, Section VI. The experimental result of the proposed methods.Section VII. Finally, the conclusion is presented

\section{II.}

\section{CONTROLLER DESIGN}

\section{A) PID controllers}

The PID has characterized by high-quality performance and simplicity in design. It has three important parts are proportional gain $\mathrm{Kp}$, integral gain $\mathrm{Ki}$, and derivative gain $\mathrm{Kd}$ so performing the controller can improve by tuning these parameters. Most PID controllers nowadays are digital [10]. The action control of PID in continuous-time is expressed in equation (1) and equation (3) is shown PID control after taking Laplace Transform for equation (1). One method to get PID control in Z-transform is substitution $S$ in equation (3) with equation (4) [11].

$$
\begin{aligned}
& \mathrm{u}(\mathrm{t})=\mathrm{Kpe}(\mathrm{t})+\mathrm{Ki} \int_{0}^{\mathrm{t}} \mathrm{e}(\mathrm{t}) \mathrm{dt}+\mathrm{Kd} \frac{\mathrm{de}}{\mathrm{dt}} \\
& e(t)=y(t)-r(t) \\
& \mathrm{PID}(\mathrm{s})=\mathrm{kp}+\mathrm{ki} \frac{1}{\mathrm{~S}}+\mathrm{kd} \mathrm{S} \\
& \mathrm{S}=\frac{2}{\mathrm{Ts}} \frac{(\mathrm{Z}-1)}{(\mathrm{Z}+1)}
\end{aligned}
$$

where $\mathrm{u}(\mathrm{t})$ is the control action, $\mathrm{e}(\mathrm{t})$ is the error signal, $\mathrm{r}(\mathrm{t})$ is the reference and $\mathrm{y}(\mathrm{t})$ is process output of the system, and $\mathrm{Ts}$ is the sampling period.

\section{B) Fuzzy Logic}

Fuzzy Logic is one method intelligent to decide such as a decision in mind human and does not require precise mathematical modeling knowledge. Lotfi Zadeh proposed the term fuzzy logic in 1965[12]. So the fuzzy logic idea is the transition from classical logic (expresses by true or false) to become multiple values between zero and one, which represents its transition to philosophical and linguistic mathematics. Figure 2 is shown a general structure of FLC. It requires the following three steps to implement Fuzzy logic technique to a real application [13]:

- Fuzzification is converting crisp data or classical data into Membership Functions (MFs) or fuzzy data.

- Fuzzy Inference Process is to incorporate membership functions with the rules of control.

- Defuzzification is converting fuzzy control into real output or real control action So that uses different methods to evaluate each associated output.

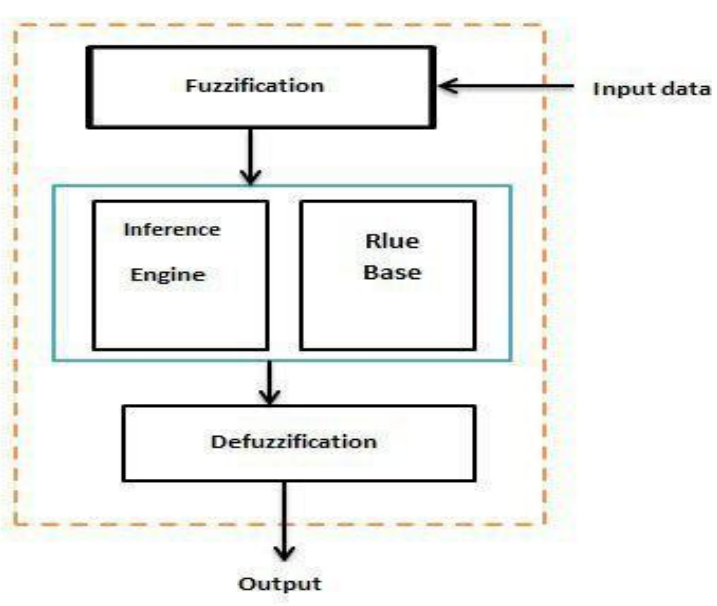

Fig. 2 General Structure of FLC [12]

Fuzzy logic control technology has seen a lot of interest in the past years for its strong performance as a controller on the system. Many publications research includes the field of design fuzzy controller to more stability system. An FLC, in particular, has a better control impact in situations of non-linear and time-varying of the system similar to a modern controller or classical controller [14]. Fuzzy logic control on our proposed design is used to an adaptive gain of the PID with a set of $\mathrm{Kp}, \mathrm{Ki}$, and $\mathrm{Kd}$ depends on error and change error so that the input of the Fuzzy logic is error and change error and the output of Fuzzy logic is the PID controller parameter. The PID controller parameter will vary with time, based on the rules of Fuzzy logic. In the first moments of the system, the error is big so Fuzzy logic will give big gain to the system. Figure. 3 is shown the Fuzzy logic adaptive gain of a PID controller. 


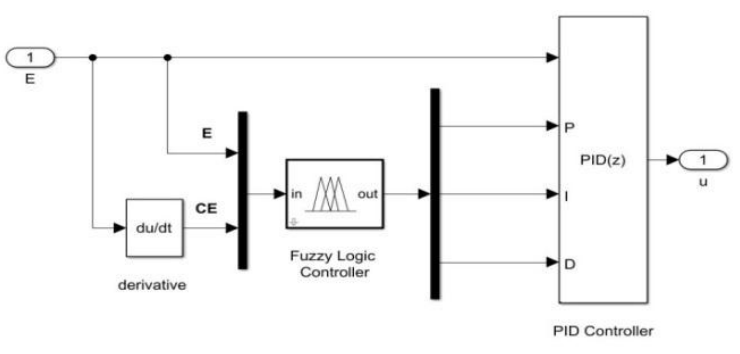

Fig. 3 the Fuzzy logic adaptive gain of a PID controller.

Figure 3 is shown the FLC that has two input variables of the controller are error (e) and derivative of error that is change error and three variables in the output $(\mathrm{Kp}, \mathrm{Ki}, \mathrm{Kd})$. the values of the $\mathrm{E}$ and $\mathrm{CE}$ in range of [-2 2], each input and output has five memberships which are NB (Negative Big), NM (Negative Medium), Z (Zero), PM (Positive Medium) and PB (Positive Big). The triangular shape memberships choose for input and output, the mamdani type in the inference process, and the centroid (center of gravity) technique that used in the Defuzzification method. More experience has been to write rules of fuzzy logic so our proposal uses PSO to find optimal rules. The number rules are number memberships power number input so at our work is twenty-five rules.

\section{C) Particle swarm optimization}

PSO is one of the most common algorithms in a population-based literature style. Developed and presented by Kennedy and Eberhart in 1995. It is inspired by the social behavior of animals such as fish schooling or birds flock. The PSO is identical to evolutionary techniques such as Genetic Algorithms (GAs). Where it initializes the system with a random population matrix and updates generations to search for the optimum solution. However, unlike the GAs, The PSO does not have any evolutionary operations like crossover and mutation. The PSO has successfully applied in many research areas. It has shown the PSO better results in a faster, cheaper compared with other methods. Another factor PSO is appealing is that it has few parameters to change. PSO was used for approaches that can use across a wide variety of applications, and for specific requirements.

In the PSO algorithm, a particle is named for each solution of a problem that can move in a search area and update the position of each particle. PSO has two important vectors are a velocity vector and a position vector. The position vector determines the value of the solutions to a problem. The position of particles is updated using the following equation (5). The velocity vector determines the intensity of movement and direction which the velocity vector is defined as (6) [15].

$$
\begin{gathered}
X i(t+1)=X i(t)+V i \\
(t+1)
\end{gathered}
$$

$$
\begin{gathered}
V i(t+1)=w V i(t)+C 1 r 1(P i(t)-X i(t)) \\
+C 2 r 2(G-X i(t))
\end{gathered}
$$

where the updated and current particle positions are $X i(t+1), X i(t)$, and the updated and current particles velocities are $V i(t+1), V i(t)$. the best solution got by the ith particle is $\operatorname{Pi}(\mathrm{t})$, and the best solution by all particles inside the swarm is $(\mathrm{G}), \mathrm{r} 1$ and $\mathrm{r} 2$ are random numbers in a range $[0,1], \mathrm{C} 1$ and $\mathrm{C} 2$ are two positive constants which control the impact of PSO global and local search capabilities and usually equal 2 .and the inertia weight is denoted $\mathrm{w}$, which has a very important role in PSO convergence behavior to control the velocity. In this paper, the parameter of PSO is used as shown in Table1. The efficiency of PSO will be improved if the inertia weight is decreased linearly from 0.9 to 0.4 by the following equation (7) [16], where Itermax is the maximum of iteration in the evolution process, Wmax and $\mathrm{Wmin}$ are values of inertia weight at maximum (0.9) and minimum (0.4), and iter is the current value of iteration [14].

$$
\mathrm{Wi}=\mathrm{Wmax}-\frac{(\mathrm{Wmax}-\mathrm{Wmin})}{\text { Itermax }} \text { iter }
$$

TABLE 1

PSO PARAMETERS

\begin{tabular}{cc}
\hline Parameters & Value \\
\hline $\mathrm{C} 1$ & 2 \\
\hline $\mathrm{C} 2$ & 2 \\
\hline $\mathrm{Wmin}$ & 0.4 \\
\hline $\mathrm{Wmax}$ & 0.8 \\
\hline Number of particle(n) & 5 \\
\hline Number of iterations (itr) & 25 \\
\hline
\end{tabular}

The fitness function of a PID controller can include performance criteria Such as the rise time (tr), the settling time ( ts), the overshoot (MP), and the steady-state error (Ess) [16]. It should be noted that the definition of fitness function may differ from researcher to another researcher, and therefore there is no systematic way to define a proper fitness function within the PSO so every problem has different fitness functions. In this paper, the fitness function is used as shown in (8) [5].

$$
J=\int_{0}^{-\infty}[w 1|e(t)|+w 4|e(t)|] d t+w 3 t s
$$

The ts of response can reduce by the term w3 ts where w3 can make the smooth of the response curve. Also, the term w4 is punishment to avoid the overshoot of response. Where w4 is much larger than w1. The choice of parameters of w1, w3, w4 is very critical to find a solution to a problem. 
In PSO, every particle has a lot of variables so every particle has seventy-five variables to represent a set of rules on fuzzy logic in this paper. Seventy-five variables are divided into three groups to organize the roles of one of the fuzzy logic outputs. The PSO -fuzzy PID works with the following steps:

1- Initialize the parameter of PSO.

2- Create a particle population randomly.

3- Change fuzzy logic rules.

4- Calculate the fitness function.

5- Calculate the best solution obtained by all particles and the current iteration.

\section{True Time Toolbox}

The term NCS is a named that combines a network system and control system. All factors have to be taken into respect when designing NCS models or evaluating a controller algorithm so the NCS operates in the cosimulation field of network and control design. The cosimulation method uses TrueTime toolbox [17]. In our work, TrueTime tools were developed at Lund University in the Department of Automatic Control in 1999 which provides a platform for designing control systems and simulating various types of networks that work in MATLAB. TrueTime tool library version 2.0 at 06-042016 is shown in the figure 4.TrueTime toolbox library includes TrueTime Kernel, TrueTime Send, TrueTime Receive, TrueTime Network (wired network), TrueTime Battery, TrueTime Ultrasound Network, and TrueTime Wireless Network. These blocks are MATLAB S-functions written in $\mathrm{C}++$, variable step, discrete [18].

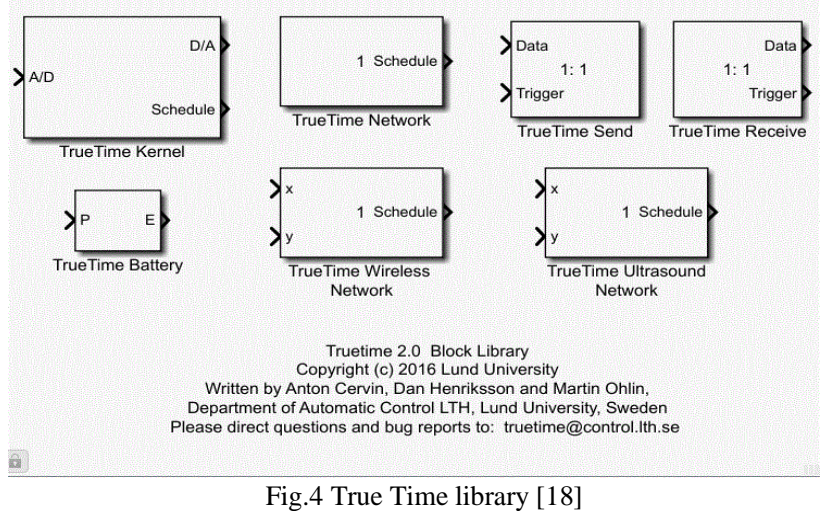

A Kernel block is used to simulate a computer node. This block is initialized by invoking some functions written using the $\mathrm{C}++$ programming language or in m.file similar to the real-time of the operating system. The True Time Kernel is responsible for performing user-defined tasks and dealing with interrupt handlers, which include network interfaces and input/output tasks, timers and control algorithms. The kernel block is allowed through the A / D and D / A input port In contact with other MATLAB blocks. There are three types of scheduling policies that are supported by True Time Kernel prioFP represents the fixed priority scheduling, prioDM represents the deadlinemonotonic scheduling, and prioEDF represents the first Earliest-deadline-first scheduling where every Kernel block can be connected to a separate local memory to sort the data for each task.

The wired network provides seven protocols: Ethernet, Flex Ray, Round Robin, Time Division Multiple Access (TDMA), Frequency Division Multiple Access (FDMA), Carrier Sense Multiple Access/Collision Detection (CSMA / CD) and Carrier Sense Multiple Access/Arbitration Message Priority (CSMA / AMP). The wireless block also works similarly to the wired block, but it needs to $\mathrm{X}$ and $\mathrm{Y}$ to assign a node. The True Time wireless network block supports WLAN (IEEE $802.11 \mathrm{~b} / \mathrm{g}$ ) and ZigBee (IEEE 802.15.4) protocol. The following steps use to establish a WNCS with of True Time Toolbox [19]:

1- Building a WNCS model in real-time using MATLAB / Simulink and True Time.

2- Create nodes by configuring a True Time Kernel block to design each node such as the controller and sensor.

3- Define parameters to set some initial values for each node with the use of $m$.file code function.

4- Determine the type of network for the design and set its parameters such as transmit power, number of nodes, packet loss probability, minimum frame, data rate, etc.

\section{IV.ZigBee Network}

ZigBee technology is one of the most important recently deployed wireless options based on 802.15.4 standards. It has many advantages including low cost, short time delay feature, low power consumption, and data rate. ZigBee covering the top layers of the protocol stack, where 802.15.4 is accountable for the layers Medium-Access Control (MAC) and the physical (PHY).The carrier sense multiple access with collision avoidance (CSMA-CA ) is used to improve the probability of successful data transmission [20]. Zigbee protocol characteristics comprise: provide for multiple topologies such as point to multipoint and point to point and mesh networks. In addition to the applications provided by ZigBee, including remote control, building automation, security systems, remote meter reading, and computer peripherals [21] Zigbee was developed to provide the following functionalities:

1- Low cost to install and maintain.

2- ZigBee device battery does not need to chargers for years.

3- An about $70 \mathrm{~m}$ indoors and $400 \mathrm{~m}$ outdoors are range ZigBee wireless network.

4- The maximum data rates for each frequency band are $20 \mathrm{kbps}$ for $868 \mathrm{MHz}, 40 \mathrm{kbps}$ for $915 \mathrm{MHz}$, and $250 \mathrm{kbps}$ for $2.4 \mathrm{GHz}$. 
Al-Furat Journal of Innovation in Electronics and Computer Engineering (FJIECE) is an international, open access, interdisciplinary, quarterly, and double blind peer-reviewed journal published by Al-Furat Al-Awsat Technical University/Iraq. It is dedicated to the latest advancement in Electronic Engineering, Computer engineering, and their related and subfields.

In this paper, True Time uses to simulate the Zigbee wireless network parameters [6] are shown in Table 2. Every node has a location in an area where $\mathrm{X}$ and $\mathrm{Y}$ determine the location of the node. The positions of interferences nodes to the sensor/actuator node studied in the experiment [21]. Sensor/Actuator node location is positioned at $(50,0)$, Controller node location is positioned at $(0,0)$ and Interference Receiver location is positioned at $(110,0)$ and Interference Sender location is positioned at $(10,0)$.

TABLE2

ZIGBEE PARAMETERS

\begin{tabular}{cc}
\hline Parameters & Value \& units \\
\hline Network Type & $802.15 .4(Z i g B e e)$ \\
\hline Network Number & 1 \\
\hline Number of Nodes & 4 \\
\hline Data Rate & $250000 \mathrm{Bps}$ \\
\hline $\begin{array}{c}\text { Minimum Frame } \\
\text { Size }\end{array}$ & $248 \mathrm{Bit}$ \\
\hline $\begin{array}{c}\text { Transmit Power } \\
\text { Receiver Signal } \\
\text { Threshold }\end{array}$ & $3 \mathrm{dBm}$ \\
\hline
\end{tabular}

\section{Simulation OF WNCS}

Figure 5 illustrates the proposed WNCS model in this work that has two parts, regulator node and sensor/actuator node, which are connected by the wireless communication network (ZigBee). The regulator node contains one A/D input and D/A output. The regulator node receives a response system via the network, then output on port D/A. The fuzzy PID process error and give action control to A/D port. The regulator sends action control via the network. The sensor/actuator node contains one A/D input and D/A output. The sensor/actuator receives an action control via the network, then output it on port D/A. The stepper motor that has the transfer function and all of the relevant parameters are taken from [23] is shown in equation (9) will run and give the response system to $\mathrm{A} / \mathrm{D}$ port. The sensor/actuator sends action control via the network. Also, two Interference blocks are used, one for the sender and one for the receiver. It aims to simulate the number of nodes loaded on the network, test the effect of time-varying delay on the number of packets, and calculate the average Round trip time RTT of transmission packets between the sensor /actuator and the regulator nodes. So, the packet size of the Interference sender node is sent. As shown in equation (10) [8]. PSO optimizer works to find the best fuzzy rules that the fuzzy will set the output to base on it so PID will read parameter (KP, KI, and $\mathrm{KD}$ ) as values are variable. with time run. Our proposal was simulating on MATLAB 2018b and True Time 2.0 in 2016.

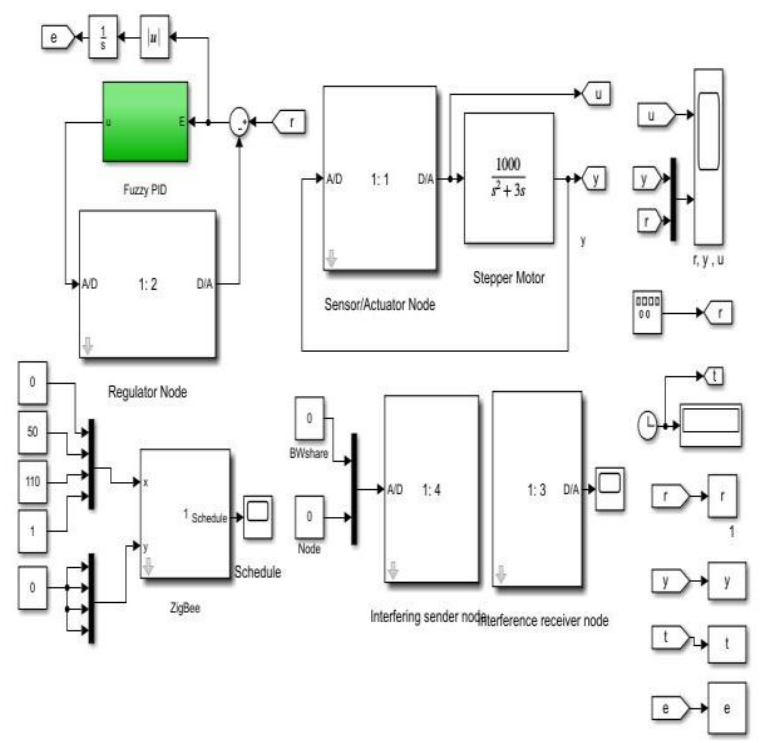

Fig.5 The proposed WNCS model

$G(s)=$
$\frac{1000}{s^{2}+3 s}$

Packet length $=($ Nodes $* \mathrm{BW}$ share $\times$ Max $\mathrm{P} \times \mathrm{W})$ bytes $(10)$

Where the maximum packet size is Max $\mathrm{P}$, and the small portion of the bandwidth is BWshare, and a weighting index of BWshare $(\mathrm{W})$ is a random number between $(0,1)[5]$.

There are two tasks in the Sensor / Actuator node called Sensor task and Actuator task. The Sensor task will be based on a time-based operation. The sensor task will sense the plant every sample time that calculates depend on Shannon's Sampling Theorem. The Sensor / Actuator node performs sensing the output of the plant, reading plant measurement, preparing the sensor packet and, and sending the sensor packet to the regulator node over the Zigbee network. The actuator task is will base on the event-based operation and performs reading the controller packet from the Zigbee.

The controller or regulator node has one task called controller task. It received the sensor signal from the Zigbee network interface, then this signal processed by Fuzzy PID. The controller task will be based on the eventbased operation and implemented by reading the sensor packet from the ZigBee network interface, obtaining a control signal, preparing the control packet, and sending a control packet over the Zigbee network. Figure 6 shows the algorithm followed by the sensor and actuator. 
Al-Furat Journal of Innovation in Electronics and Computer Engineering (FJIECE) is an international, open access, interdisciplinary, quarterly, and double blind peer-reviewed journal published by Al-Furat Al-Awsat Technical University/Iraq. It is dedicated to the latest advancement in Electronic Engineering, Computer engineering, and their related and subfields.

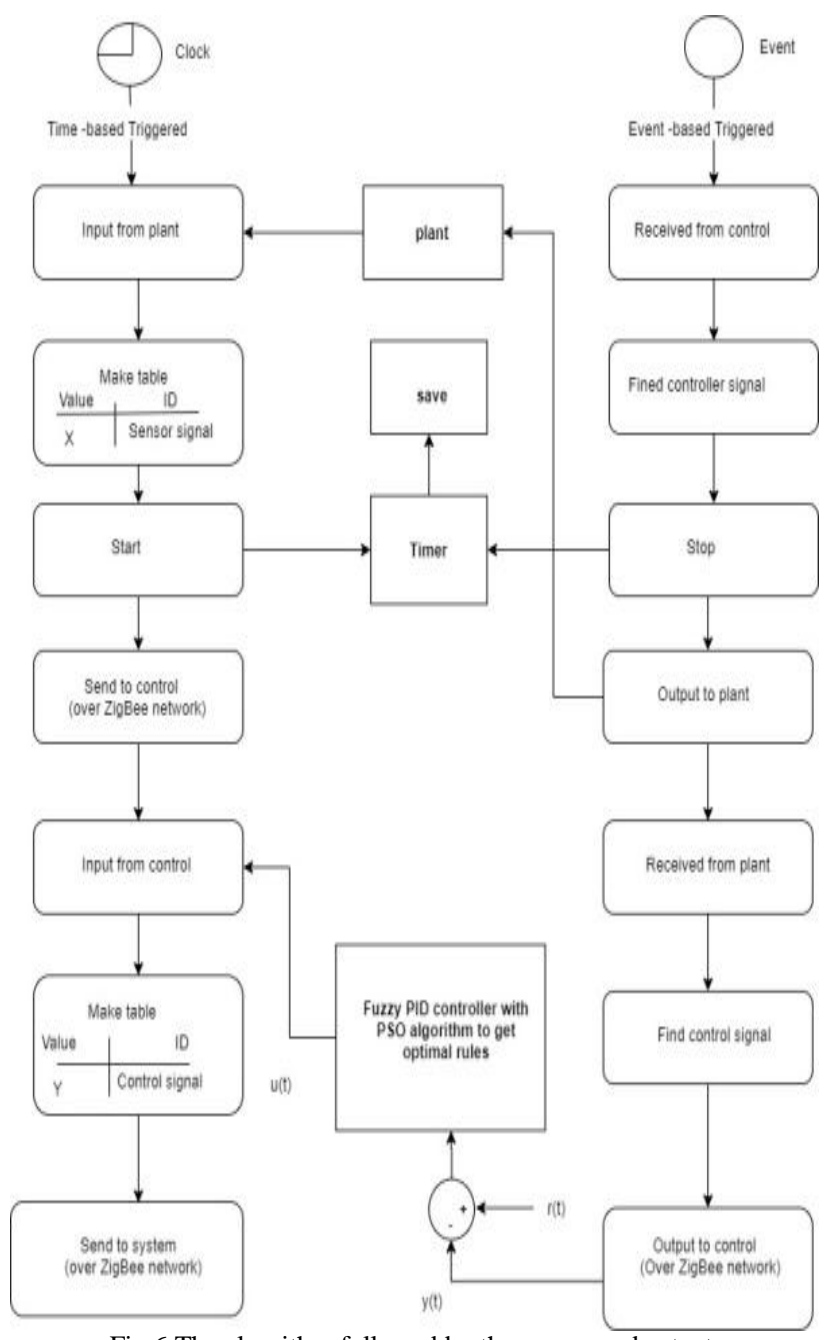

Fig.6 The algorithm followed by the sensor and actuator.

\section{RESULT}

In our work, 40 minutes simulate for the WNCS model proposed without load. The sample time is equal to 0.08 sec based on Shannon's theory of samples to control the speed of the stepper motor. The input voltage for a stepper motor is between +1 and -1 to rotate the stepper on clockwise or counter wise. Figure 7 shows the system response and action control with a no-load network ( nodes $=0$ and $\mathrm{BW}$ share $=0$ ).
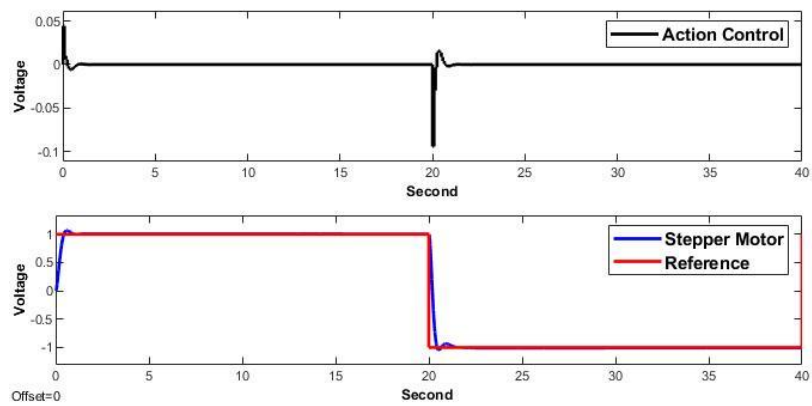

Fig.7 System Response for Fuzzy PID Controller for Ts $=0.08 \mathrm{~s}$, with no Load
For $40 \mathrm{sec}$ simulation time, the RTT values are shown in figure 8, and the histogram of this RTT is shown in Figure 9. The number of Packets: 467, the average of RTT= $0.0214 \mathrm{sec}$ and Overshoot: $6.5684 \%$.

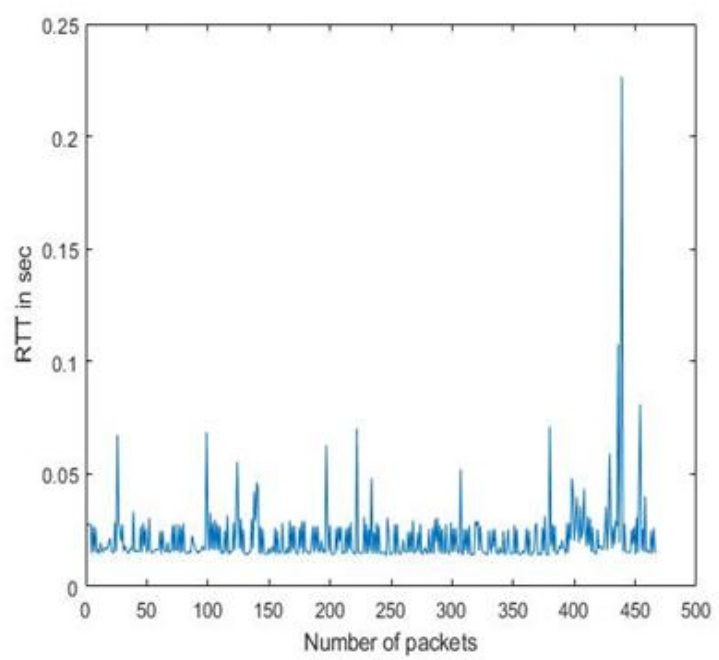

Fig.8. RTT Histogram for Fuzzy PID Controller for Ts=0.08s (with no Load network).

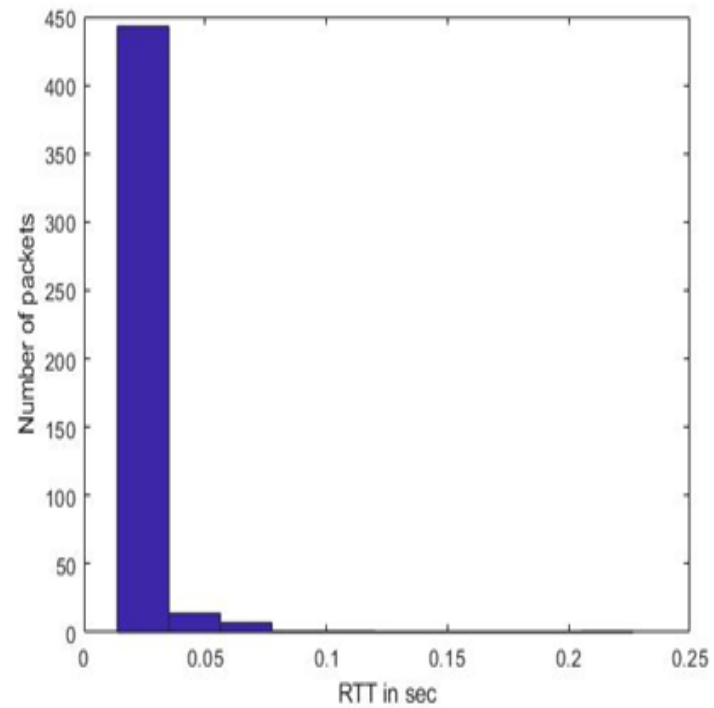

Fig. 9 The number of a packet with RTT for Fuzzy PID Controller for $\mathrm{Ts}=0.08 \mathrm{~s}$ (with no Load network).

In the table (3),(4) shows three levels of sampling time will be tested until system stability is reached [0.035, 0.5, 1] seconds and using a different number of nodes. And study performance criteria of a system such as a rise time ( tr), the settling time( ts), the overshoot (MP). With two scenarios (Medium loaded network (BWshare 0.4) at scenario one, and High loaded network (BWshare 0.9) at scenario two). 
Al-Furat Journal of Innovation in Electronics and Computer Engineering (FJIECE) is an international, open access, interdisciplinary, quarterly, and double blind peer-reviewed journal published by Al-Furat Al-Awsat Technical University/Iraq. It is dedicated to the latest advancement in Electronic Engineering, Computer engineering, and their related and subfields.

TABLE 3

WNCS MEDIUM LOADED NETWORK AT BWSHARE 0.4

\begin{tabular}{|c|c|c|c|c|}
\hline \multirow{3}{*}{$\begin{array}{l}\text { Sampling } \\
\text { Time in } \\
\text { Interference } \\
\text { Sender node }\end{array}$} & \multicolumn{4}{|c|}{$\begin{array}{l}\text { Medium Loaded network at BWshare } \\
\text { 0.4 scenario one }\end{array}$} \\
\hline & \multicolumn{4}{|c|}{$\begin{array}{c}\text { sampling time of the system is } 0.08 \\
\text { sec }\end{array}$} \\
\hline & $\begin{array}{l}\text { N. of } \\
\text { Node }\end{array}$ & МР\% & TS & TR \\
\hline 0.035 & 15 & 0.8638 & 1.4653 & 0.6189 \\
\hline 0.5 & 210 & 0.9776 & 1.5616 & 0.6438 \\
\hline 1 & 500 & 1.4022 & 1.8228 & 0.6358 \\
\hline
\end{tabular}

TABLE 4

WNCS WITH HIGH LOADED NETWORK AT BWSHARE 0.4

\begin{tabular}{lcccc}
\hline $\begin{array}{l}\text { Sampling } \\
\text { Time in } \\
\text { Interference }\end{array}$ & \multicolumn{4}{c}{$\begin{array}{c}\text { High Loaded network at BWshare } \\
\text { 0.4 scenario two }\end{array}$} \\
\cline { 2 - 5 } $\begin{array}{l}\text { Sender } \\
\text { node }\end{array}$ & \multicolumn{4}{c}{ sampling time of the system is 0.08 sec } \\
\cline { 2 - 5 } & $\begin{array}{c}\text { N. of } \\
\text { Node }\end{array}$ & MP\% & TS & TR \\
\hline 0.035 & 10 & 0.8671 & 1.4601 & 0.5455 \\
\hline 0.5 & 120 & 0.8600 & 1.5593 & 0.5474 \\
\hline 1 & 300 & 1.8264 & 2.0885 & 0.6288 \\
\hline
\end{tabular}

The three nodes contain two control system nodes (Sensor/Actuator, Controller nodes) and(interference receiver node) with many nodes is set to 500 nodes that emulate by Interference sender node so the total nodes in WNCS are equal to 503 nodes .a packet length describes in equation $(10)$ will be $(503 \times 0.4 \times 133 \times \mathrm{w})$ byte. In addition to the controller packet and sensor packet, other packets will be sent via the wireless network. The system response stays stable with 503 nodes. Figure 10 shows system response for fuzzy PID controller and action control for $\mathrm{Ts}=0.08 \mathrm{~s}$, with medium load, BW share 0.4 and 500 nodes.
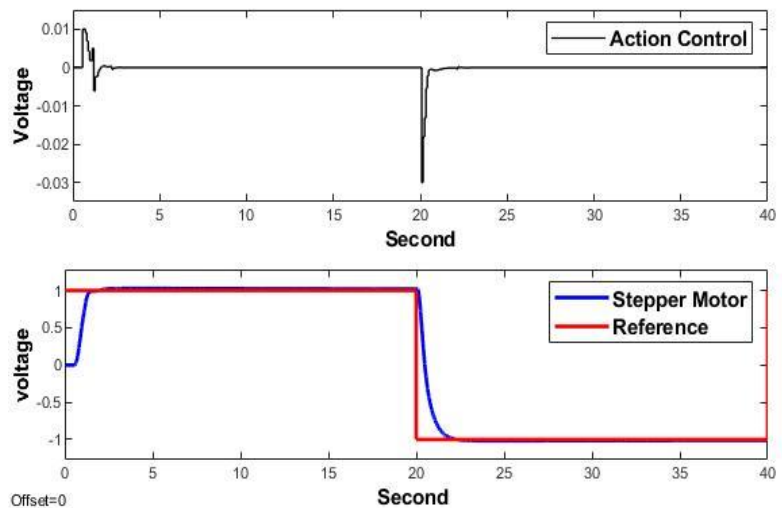

Fig.10 System Response for Fuzzy PID Controller and action control with medium Load BWshare 0.4 and 500 nodes
After the PSO algorithm selected the optimal rules for fuzzy control. A fuzzy Logic adaptive gain of the PID controller, automatically. Variable values are obtained for the three gain parameters $\mathrm{Kp}, \mathrm{Ki}, \mathrm{Kd}$. Figure 11 shows the variable gain values for the parameters of the PID controller are with a time of the simulation.

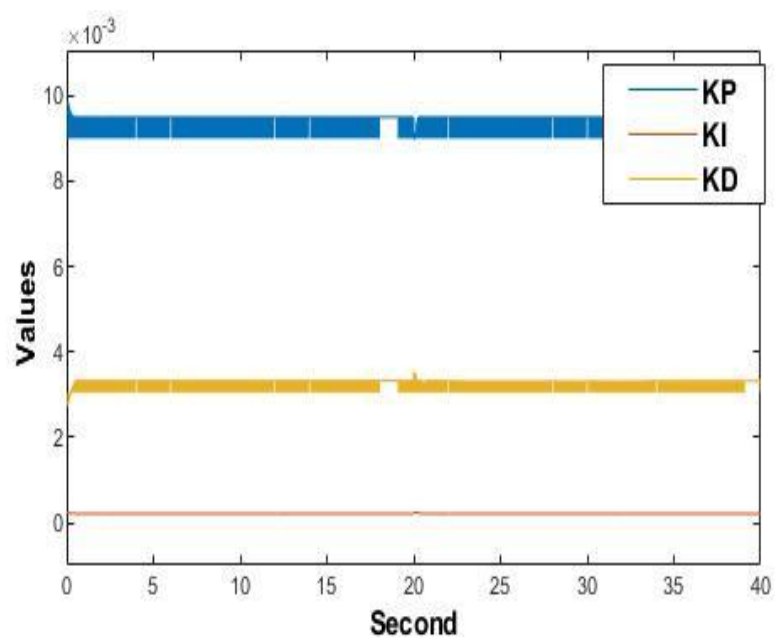

Fig.11 Gain values for the parameters of the PID controller with a time of the simulation

\section{CONCLUSION}

For a WNCS design proposed. Most of the major problems facing this system must be taken into consideration. One of these problems is various time delays that have an impact on the performance and stability of the system. These problems are eliminated by designing a robust controller that handles the load on the network, especially when the number of nodes increases.

In this paper, the Fuzzy PID controller is suggested to control the stepper motor wirelessly and using the PSO algorithm to find the optimal rules. This controller is tested when sampling time of the system is $0.08 \mathrm{sec}$ while the sampling time in the interfering sender node is one sec, which can be handle 300 and 500 nodes when BWshare is 0.9 and 0.4 respectively, used Zigbee because it reaches more areas of the network compared to Wi-Fi. Despite the results achieved above, more improvements are still needed.

\section{NOMENCLATURE}

e(t) System Error

$\mathrm{u}(\mathrm{t}) \quad$ Output of the controller

G(s) Transfer Function of plant

C1 Cognitive Learning Factor

C2 Social Learning Factor 
Al-Furat Journal of Innovation in Electronics and Computer Engineering (FJIECE) is an international, open access, interdisciplinary, quarterly, and double blind peer-reviewed journal published by Al-Furat Al-Awsat Technical University/Iraq. It is dedicated to the latest advancement in Electronic Engineering, Computer engineering, and their related and subfields.

Kd Derivative Gain

$\mathrm{Ki} \quad$ Integral Gain

Kp Proportional Gain

W Inertia Weight

r(t) Input Signal

$y(t) \quad$ Output of The System

$\mathrm{Pi} \quad$ Is the best solution of Ith particle

\section{ACKNOWLEDGMENT}

I would like to extend my thanks and gratitude to the teaching staff in the Department of Communication Engineering Technical/ Al Najaf and my special gratitude to my supervisor (Ahmad T. Abdulsadda) who helped me to do this research paper on the topic

(Adaptive Gain Scheduling Based Controller Networking Control Systems) It also helped me in identifying a lot of research.

\section{REFERENCES}

[1] Marie, Mehdi J., Ghaida A. Al-Suhail, and Salah Al-Majeed. "Client-server based wireless networked control system." 2016 IEEE East-West Design \& Test Symposium (EWDTS). IEEE, 2016

[2] Guo, Lejiang, et al. "The theory and architecture of network control system." 2010 International Conference on Intelligent Computing and Cognitive Informatics. IEEE, 2010.

[3] Tang, Xiao-Ming, and Bao-Cang Ding. "Design of networked control systems with bounded arbitrary time delays." International Journal of Automation and Computing 9.2 (2012): 182-190.

[4] Zhang, Dan, et al. "Analysis and synthesis of networked control systems: A survey of recent advances and challenges." ISA transactions 66 (2017): 376-392.

[5] Abdul-Hussain, Russul N., and Osama A. Awad. "Studying The Effect of Sampling Time and Network Load on Wireless Networked Control Systems." Journal of Al-Qadisiyah for computer science and mathematics 11.3 (2019): Page-31.

[6] Salman, Mais M., and O. A. Awad. "Evaluating a ZigBee Network with SMC for Hard and Concurrent Parameter Variations.",2017

[7] Deng, Jian-Qiu, et al. "Research on gain scheduling controller of the networked control system with long delay." International Journal of Control, Automation and Systems 13.1 (2015): 33-38.

[8] Aung, N., et al. "Simulation of Wireless Networked Control System Using TrueTime And Matlab." International Journal of Scientific and Technology Research 6.5 (2015): 31-35.

[9] Awad, Osama A., and Israa Laith. "Gain Scheduling Fuzzy PID Controller for Distributed Control Systems." International Conference on Applied Computing to Support Industry: Innovation and Technology. Springer, Cham, 2015.

[10] Abramovitch, Daniel Y. "A unified framework for analog and digital PID controllers." 2015 IEEE Conference on Control Applications (CCA). IEEE, 2015.

[11] Starr, Gregory P. "Introduction to applied digital control." Lecture Notes in Digital Control (2006): 5-31.

[12] Czabanski, Robert, Michal Jezewski, and Jacek Leski. "Introduction to fuzzy systems." Theory and Applications of Ordered Fuzzy Numbers. Springer, Cham, 2017. 23-43.
[13] Jones, André, Arnold Kaufmann, and Hans-Jürgen Zimmermann, eds. Fuzzy sets theory and applications. Vol. 177. Springer Science \& Business Media, 2012.

[14] Zadeh, Lotfi A., King-Sun Fu, and Kokichi Tanaka, eds. Fuzzy sets and their applications to cognitive and decision processes Proceedings of the us-japan seminar on fuzzy sets and their applications, held at the university of california, berkeley, california, july 1-4, 1974. Academic press, 2014.

[15] Tharwat, Alaa, et al. "Particle swarm optimization: a tutorial." Handbook of research on machine learning innovations and trends. IGI global, 2017. 614-635..

[16] Li, Xu-zhou, Fei Yu, and You-bo Wang. "PSO algorithm based online self-tuning of PID controller." 2007 International Conference on Computational Intelligence and Security (CIS 2007). IEEE, 2007.

[17] Ogata, Katsuhiko, and Yanjuan Yang. Modern control engineering. Vol. 5. Upper Saddle River, NJ: Prentice hall, 2010.

[18] Cervin, Anton, Dan Henriksson, and Martin Ohlin. "TrueTime 2.0 beta-Reference manual." Department of Automatic Control, Lund University (2010): 21-25.

[19] Wanga, Y., and L. Heb. "Analysis and Simulation of Networked Control Systems Delay Characteristics Based on Truetime." Computer Modelling and New Technologies 17.4 (2013): 210-216.

[20] Song, Chau-Chung, et al. "Simulation and experimental analysis of a ZigBee sensor network with fault detection and reconfiguration mechanism." 2011 8th Asian Control Conference (ASCC). IEEE, 2011.

[21] Hwang, Soyoung, and Donghui Yu. "Remote monitoring and controlling system based on ZigBee networks." International Journal of Software Engineering and Its Applications 6.3 (2012) $35-42$.

[22] Cuenca, Ángel, et al. "A delay-dependent dual-rate PID controller over an ethernet network." IEEE Transactions on Industria Informatics 7.1 (2010): 18-29.

[23] Peng, Chen, Dong Yue, and Min-Rui Fei. "A higher energyefficient sampling scheme for networked control systems over IEEE 802.15. 4 wireless networks." IEEE Transactions on Industrial Informatics 12.5 (2015): 1766-1774 